\title{
Simulation of the different position on the E-type round diaphragm and the length of the beam,
}

\author{
Ahmed Jaber Abougarir, \\ Tripoli university, \\ Adel A M Abosdel \\ singhania university
}

\begin{abstract}
:
This paper studies a resonant silicon sensor whose elementary sensing component is the E-type round diaphragm, and the final sensing component is the silicon beam resonator is attached to the E-type round diaphragm. the relationship between the basic natural frequency of the beam resonator and the measured acceleration are calculated and simulated by using the above finite element modeling in detail. Because of the fact that beam is located on the different position on the E-type round diaphragm and the length of the beam, the outer radius of the E-type round diaphragm are varied respectively. The related analyses and investigations are carried out. Some important results on the relationship between the basic natural frequency of the beam resonator and the measured acceleration are obtained. Finally, based on the differential output scheme of the resonant sensor and some related criteria, a set of appropriate parameters for the above sensing structure of the sensor is determined for measuring the acceleration.

Key Words: acceleration, beam, resonator, E-type round diaphragm, micro sensor, frequency, finite element method (FEM)
\end{abstract}

\section{Introduction}

Silicone resonant sensors have developed rapidly in past decade. Many experts in sensor paid special attention to this technology.

The main advantage of the silicon microstructure resonant sensors is the advantage of generalized resonant sensors, such as direct digital output (without A/D converters), because the Sensors whose output is in the form of variable frequency are called quasi-digital because it is very easy to obtain a digital output from them. The output of digital sensors is in the form of discrete steps or states. The output of digital sensors does not require an Analogue to Digital convector and their output is easier to transmit than analog sensors. The output of digital sensors is also more repeatable, reliable, and has long-term stability. They have low hysterics and high repeatability

which is often more accurate and precise .The advantages of silicon material is that they have excellent mechanical properties, posses high strength, no mechanical hysterics. Moreover they have ability to batch process at low cost and the compatibility of mechanical and electrical properties. Meanwhile, due to their high working frequency, the dynamic characteristics of silicon resonant sensors are much better than those of conventional resonant sensors.[1]

\section{Structural Sketch and Sensing Mechanism of Microstructure Silicon Accelerometer}

Fig.1 shows the structural sketch of a microstructure silicon resonant sensor for measuring acceleration. Fig 3.2 shows the mathematical model of the sensing structure. The preliminary sensing unit of the microstructure silicon accelerometer micro-sensor for measuring the acceleration is an E-type round diaphragm, where $\mathbf{A}$ is the outer radius, $\mathbf{B}$ is the inner radius, and $\mathbf{H}$ is the thickness of the diaphragm, respectively. .

The measured acceleration within $(-100,+100) \mathrm{m} / \mathrm{s}^{2} \quad(\mathrm{a}<0 \mathrm{~F}>0, \mathrm{a}>0 \mathrm{~F}<0)$ acts perpendicularly to the lower plane of the E-type round diaphragm and yields the radial and circular stresses of the diaphragm. The final sensing unit is one pair of beams (resonator beam1 and beam 2: $\boldsymbol{L}$ is the length of the beem, $\mathbf{b}$ is the width of the beem, and $\mathbf{h}$ is the thickness of the beem, see Fig. 2) which are attached to the upper plane of the E-type round diaphragm. The location of the beam 1 and beam 2 are along the radial direction of the E-type round diaphragm. Beam 1 is located at the outer edge and beam 2 at the inner edge.

As compared with the round or square diaphragm, the E-type round diaphragm is one of the advanced elementary sensing units in silicon microstructure resonant sensors. Its major advantages are such that the stress concentrations on the effective annular sensing area, flexible stress distribution designing, easier to achieve the differential measuring scheme in order to increase the sensitivity and reduce some disturbances such as temperature, random vibration, etc. In addition, the advantage of the above sensing structure is that the beam resonator of very high $\mathrm{Q}$ factor because they can be packaged within a vacuum housing. 
The location of beam 1 and beam 2 are along the radial direction of the E-type round diaphragm (see Fig.1). beaml is located at the outer edge $r \in(A-L \sim A)$, and beam2 at the inner edge $r \in(B \sim B+L)$. However, it is very important for the silicon resonant sensor to design and select the optimal parameters of the sensing structure to measure the different acceleration ranges. Based on the sensing mechanism of the sensor, the FEM model of the sensing structure is established in detail, in this Chapter in the first step.[2]

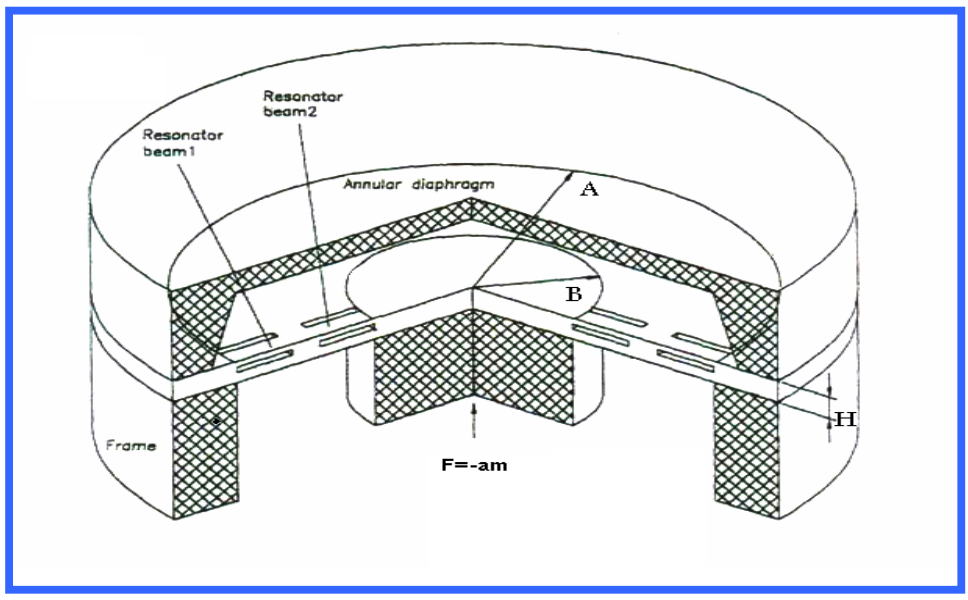

Fig 1 The structural sketch of a silicon resonant sensor for measuring acceleration

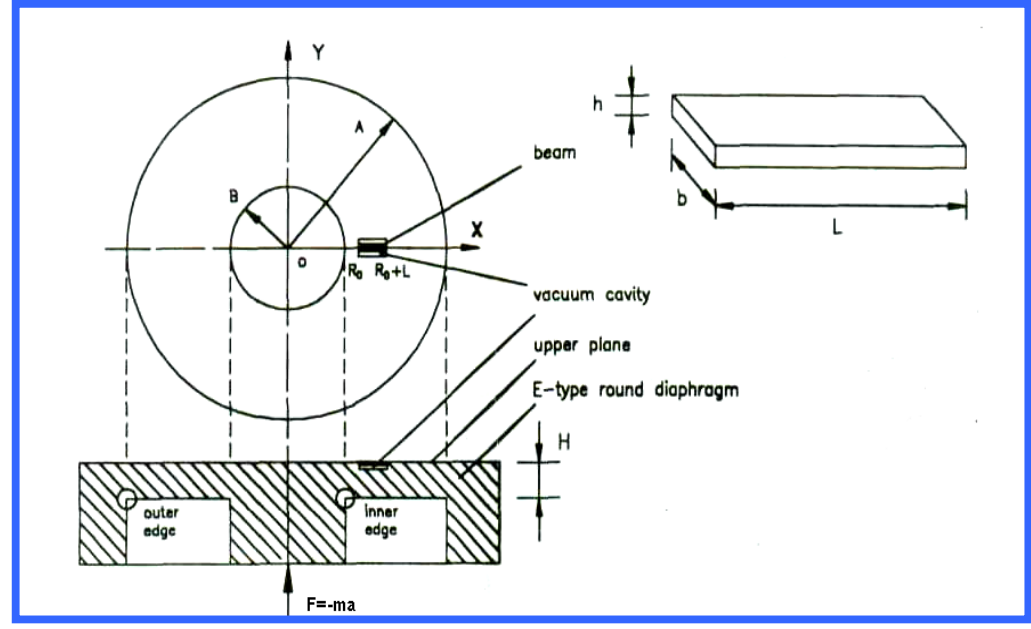

Fig 2 The mathematical model of sensing structure

III. Stresses on the upper plane of the E-type round diaphragm

The differential equation of the round diaphragm which is measuring by a acceleration can be written as following:

$$
\begin{gathered}
\frac{d}{d r}\left[\frac{1}{r} \frac{d}{d r}\left(r \frac{d w}{d r}\right)\right]=\frac{Q}{D} \\
D=\frac{E H}{12\left(1-\mu^{2}\right)} \quad Q=\frac{F}{2 \pi r} \\
F=-m a \quad m \approx \rho \pi\left(H_{S} B^{2}+H A^{2}\right)
\end{gathered}
$$

Where $\mathrm{W}$ is the normal displacement of the E-type round diaphragm, $\mathrm{D}$ is the flexural rigidity of the diaphragm, $\mathrm{Q}$ is the shearing force of the circle of radius $r$ of the diaphragm, $r$ is radial coordinate of the diaphragm in polar coordinate, $\mathrm{F}$ is the inertial force caused by the measured acceleration a , $\mathrm{m}$ is the effective sensing mass, $H_{S}$ is the height of the sensing cylinder and $\mathrm{E}, \rho, \mu$ are Young's modulus density and Poisson ratio of the sensing 
structure, respectively. It should be pointed out that the positive direction of the measured acceleration a and the corresponding inertial forces are shown in fig 2 . If the amount of the above both variables is appositive, the actual direction of the above variable is the same with that in fig 2 . And if the amount of the above both variables is a negative, the actual direction of the above variable is opposite from that in fig.2.[4]

The boundary conditions at the inner and outer edges of the E-type diaphragm are as follows:

$$
\begin{cases}r=B & \frac{d w}{d r}=0 \\ r=A & w=\frac{d w}{d r}=0\end{cases}
$$

From equation (3.1) and (3.2), the normal displacement of the E-type round diaphragm $\mathrm{w}$ can be obtained. Then the radial stress $\sigma_{r}(r)$ and circular stress $\sigma_{\theta}(r)$ of the E-type round diaphragm can be obtained as follows :

$$
\begin{aligned}
& \left\{\begin{array}{l}
\sigma_{r}(r)=\frac{-3 F}{4 \pi H^{2}}\left[\left(2 \ln R+2-C-\frac{C}{R^{2}}\right)+\left(2 \ln R-C+\frac{C}{R^{2}}\right) \mu\right] \\
\sigma_{\theta}(r)=\frac{-3 F}{4 \pi H^{2}}\left[\left(2 \ln R+2-C-\frac{C}{R^{2}}\right) \mu+\left(2 \ln R-C+\frac{C}{R^{2}}\right)\right]
\end{array}\right. \\
& K=\frac{B}{A} \quad C=\frac{-2 K^{2} \ln K}{1-K} \quad R=\frac{r}{A}
\end{aligned}
$$

\subsection{Reckoning Model of the Beam}

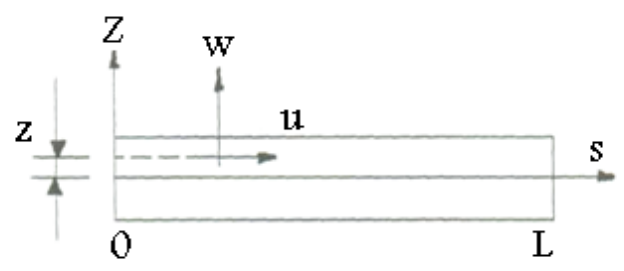

Fig 3 The mathematical model of the beam

Fig 3 shows the mathematical model of the beam. The axial and normal vibrating displacements $\mathrm{u}(\mathrm{s}, \mathrm{z}, \mathrm{t})$ and $\mathrm{w}(\mathrm{s}, \mathrm{t})$ of beam at an arbitrary point in Cartesian coordinate can be written as:

$$
\left\{\begin{array}{l}
u(s, z, t)=-z \frac{d w(s)}{d s} \cos \omega t \\
w(s, t)=w(s) \cos \omega t
\end{array}\right.
$$

Where s, $\mathrm{z}$ are axial and normal coordinates of the beam in Cartesian coordinate, $\boldsymbol{\omega}[\mathrm{rad} / \mathrm{s}], \mathbf{w}(\mathbf{s})$ are natural frequency and its corresponding vibrating shape along the axial direction of the beam.

Energy expressions of the beam resonator are as follows :

The potential energy

$$
U=\frac{1}{2} \iiint_{V} \sigma_{S}(s, z, t) \varepsilon_{S}(s, z, t) d v
$$




$$
=\frac{E b h^{2} \cos ^{2} \omega t}{24} \int_{S}\left[\frac{d^{2} w(s)}{d s^{2}}\right]^{2} d s
$$

Where $\mathrm{S}$ is the integrated length of the beam.

The kinetic energy

$$
\begin{aligned}
T & =\frac{1}{2} \iiint_{V}\left\{\left[\frac{\partial u(s, z, t)}{\partial t}\right]^{2}+\left[\frac{\partial w(s, t)}{\partial t}\right]^{2}\right\} \rho d v \\
& =\frac{\rho b h \omega^{2} \sin ^{2} \omega t}{2} \int_{S}[w(s)]^{2} d s
\end{aligned}
$$

Where $\rho$ is density of the sensing structure.

In addition, the initial potential energy of the beam, which is caused by $\sigma_{s}^{0}(s)$, is

$$
\begin{aligned}
U_{o} & =-\frac{1}{2} \iiint_{V} \sigma_{S}^{O}(s)\left\{\left[\frac{\partial u(s, z, t)}{\partial s}\right]^{2}+\left[\frac{\partial w(s, t)}{\partial s}\right]^{2}\right\} d v \\
& =-\frac{b h \cos ^{2} \omega t}{2} \int_{S} \sigma_{S}^{O}(s)\left[\frac{d w(s)}{d s}\right]^{2} d s
\end{aligned}
$$

From fig 3.2 and equation (3.3), according to the above analyses,

$$
\sigma_{s}^{o}(s)=\sigma_{r}\left(R_{o}+s\right)=\frac{-3 F}{4 \pi H^{2}}\left\{\begin{array}{l}
{\left[2 \ln \left(\frac{R_{o}+s}{A}\right)+2-C-\frac{C A^{2}}{\left(R_{o}+s\right)^{2}}\right]} \\
+\left[2 \ln \left(\frac{R_{o}+s}{A}\right)-C+\frac{C A^{2}}{\left(R_{o}+s\right)^{2}}\right] \mu
\end{array}\right\}
$$

Then the total potential energy of the beam is

$$
U_{T}=U-U_{o}
$$

In equation (3.7) if $\sigma_{s}^{0}(s)$ is a constant, we can directly obtain the analytic relationship between the frequency and the initial axial stress can be directly obtained from equation (3.10). In this thesis, we present the FEM equation to solve the relationship between the natural frequency of the beam resonator and the measured acceleration.

$$
\omega=\frac{4.73^{2} h}{L^{2}}\left(\frac{E}{12 \rho}\right)^{0.5}\left(1+0.295 \frac{\sigma_{s}^{0} L^{2}}{E h^{2}}\right)^{0.5} \quad[\mathrm{rad} / \mathrm{s}]
$$


Beside on the above related equation we can divide the element along the axial direction of the beam (Fig 3.4, N is the total number of the beam element.) Introducing the dimensionless length: $\chi=\left(s-S_{j}\right) / \boldsymbol{l}$, $\boldsymbol{l}=0.5\left(S_{j+1}-S_{j}\right)$. Then map of domain $s \in\left[S_{j}, S_{j+1}\right]$ is $\chi \in[-1,+1]$. For the displacement of the jth element, introducing the second order Hermite interpolation.

$w_{j}(s)=w_{j}(x)=X_{2}^{O} G_{2} \mathbf{a}_{j}$

where

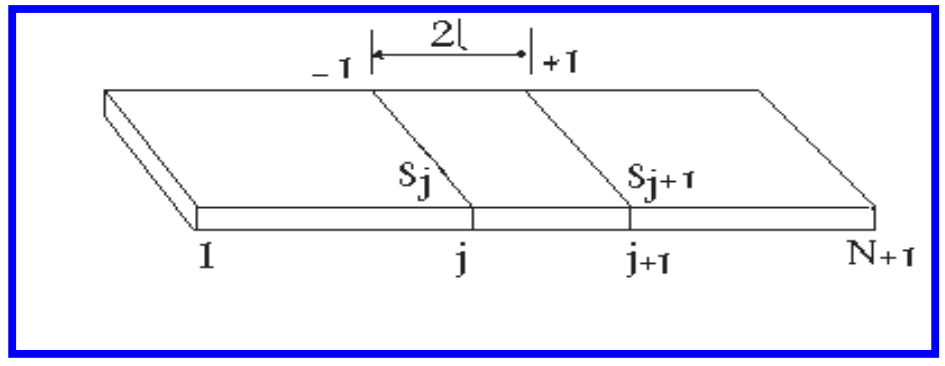

Fig 4 Dividing element along the beams axial direction

$$
\begin{aligned}
& X_{2}^{O}=\left[\begin{array}{llllll}
1 & \chi & \chi^{2} & \chi^{3} & \chi^{4} & \chi^{5}
\end{array}\right]
\end{aligned}
$$

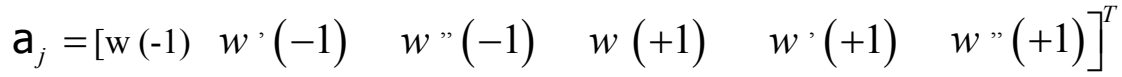

$$
\begin{aligned}
& G_{2}=\frac{1}{16}\left[\begin{array}{cccccc}
8 & 5 \imath & \iota^{2} & 8 & -5 \imath & \imath^{2} \\
-15 & -7 \imath & -l^{2} & 15 & -7 \imath & \imath^{2} \\
0 & -6 \imath & -2 \iota^{2} & 0 & 6 \imath & -2 \iota^{2} \\
10 & 10 \imath & 2 \iota^{2} & -10 & 10 \imath & -2 \iota^{2} \\
0 & \imath & \iota^{2} & 0 & -\imath & \iota^{2} \\
-3 & -3 \imath & -l^{2} & 3 & -3 \imath & \iota^{2}
\end{array}\right]
\end{aligned}
$$

From equation (3.5) (3.9),(3.11) we can obtain the element potential energy, kinetic energy and initial potential energy caused by the initial stress, in the domain $s \in\left[S_{j}, S_{j+1}\right]$ :

$$
\begin{gathered}
U^{j}=\frac{E b h^{3} \cos ^{2} \omega t}{24 \iota^{3}} \int_{-1}^{+1} \mathrm{a}_{j}{ }^{T} G_{2}{ }^{T} X_{2}{ }^{2 T} X_{2}{ }_{2} G_{2} \mathrm{a}_{j} d x \\
X_{2}^{2}=\frac{d^{2}}{d x^{2}} X_{2}^{o}
\end{gathered}
$$

$T^{j}=\frac{\rho b h \imath \omega^{2} \sin ^{2} \omega t}{2} \int_{-1}^{+1} \mathrm{a}_{j}{ }^{T} G_{2}{ }^{T} X_{2}^{o T} X_{2}^{o} G_{2} \mathrm{a}_{j} d x$

$U_{o}^{j}=-\frac{b h \cos ^{2} \omega t}{2 \imath} \int_{-1}^{+1} \sigma_{s}^{o}\left[S_{j}+(\chi+1) l\right] \mathrm{a}_{j}{ }^{T} G_{2}{ }^{T} X_{2}^{1 T} X_{2}^{1} G_{2} \mathrm{a}_{j} d x$,

$X_{2}^{1}=\frac{d}{d x} X_{2}^{o}$ 
Then we obtain the element stiffness matrix:

$$
K^{j}=\frac{E b h^{3}}{12 \iota^{3}} \int_{-1}^{+1} G_{2}{ }^{T} X_{2}^{2 T} X_{2}^{2} G_{2} d x
$$

The element mass matrix:

$$
M^{j}=\rho b h i \int_{-1}^{+1} G_{2}^{T} X_{2}^{o T} X_{2}^{o} G_{2} d x
$$

The element initial stiffness matrix:

$$
K_{o}^{j}=\frac{b h}{\imath} \int_{-1}^{+1} \sigma_{s}^{o}\left[S_{j}+(x+1) \imath\right] G_{2}{ }^{T} X_{2}^{1 T} X_{2}^{1} G_{2} d x
$$

The element total stiffness matrix:

$$
K_{T}^{j}=K^{j}+K_{o}^{j}
$$

From equation (3.18) and (3.16), we can obtain the assembly stiffness matrix $\mathrm{K}$ and assembly mass matrix $\mathrm{M}$ in the local domain $s \in[0, \mathrm{~L}]$. Then the finite element equation is the follow:

$$
\left(K-\omega^{2} M\right) \mathrm{a}=0
$$

Where the assembly nodal vector a consists of all $\mathrm{a}_{j}$.

For actual structural feature of fig 3.2, the boundary conditions of beam are as follows:

$$
\begin{cases}s=0: & w(s)=w^{\prime}(s)=0 \\ s=\mathrm{L}: & w(s)=w^{\prime}(s)=0\end{cases}
$$

From equation (3.19) and (3.20), we can obtain the natural frequency and the corresponding vibrating shapes.[5,6,7]

\section{CALCULATIONS THE LOCATION OF THE BEAM}

the main investigations are the varying laws of the measured acceleration -frequency relationship for the beam resonator as the thickness $H$, outer radius $A$ for the E-type round diaphragm and the corresponding length of the beam $L$ are varied accordingly. In order to get some generalized results of the measured acceleration frequency relationship for the beam resonator, some related parameters are selected follows:

Referenced value for the inner radius of the E-type round diaphragm is as $B=1.4 \times 10^{-3} \mathrm{~m}$

Referenced values for the width and the thickness of the beam are as $b=50 \times 10^{-6} \mathrm{~m}$ and $h=5 \times 10^{-6} \mathrm{~m}$. And the thickness of the E-type round diaphragm is as $\mathrm{H}=30 \times 10^{-6} \mathrm{~m}$

The sensor is made of silicon, $E=1.3 \times 10^{11} \mathrm{pa}, \rho=2.33 \times 10^{3} \mathrm{~kg} / \mathrm{m}^{3}, \mu=0.17$.

Basic Natural Frequency for zero acceleration $a=0$

Table 1and fig 5 shows the basic natural frequency $f(0)$ of beam with the measured zero acceleration a $=0$. From this Table and fig, we can get results: the basic natural frequency of the beam, for the zero acceleration $\mathrm{a}=0$, decreases with increasing the length of the beam $\mathrm{L}$ as the inner radius of the E-type round diaphragm $\mathrm{B}$ is a constant. 
Simulation of the different position on the E-type round diaphragm and the length of the beam,

Table 1 the basic natural frequency of the beam with $\mathrm{a}=0$ for the different $\mathrm{L}$

\begin{tabular}{|l|c|c|c|c|c|}
\hline $\mathrm{L} \times 10^{-3} \mathrm{~m}$ & $\mathbf{0 . 4 0}$ & $\mathbf{0 . 4 5}$ & $\mathbf{0 . 5 0}$ & $\mathbf{0 . 5 5}$ & $\mathbf{0 . 6 0}$ \\
\hline$f(\mathbf{0})(\mathrm{Hz})$ & $\mathbf{2 4 4 7 4 5}$ & $\mathbf{1 8 9 1 3 4}$ & $\mathbf{1 5 3 5 6 1}$ & $\mathbf{1 2 6 9 1 4}$ & $\mathbf{1 0 6 6 4 8}$ \\
\hline
\end{tabular}

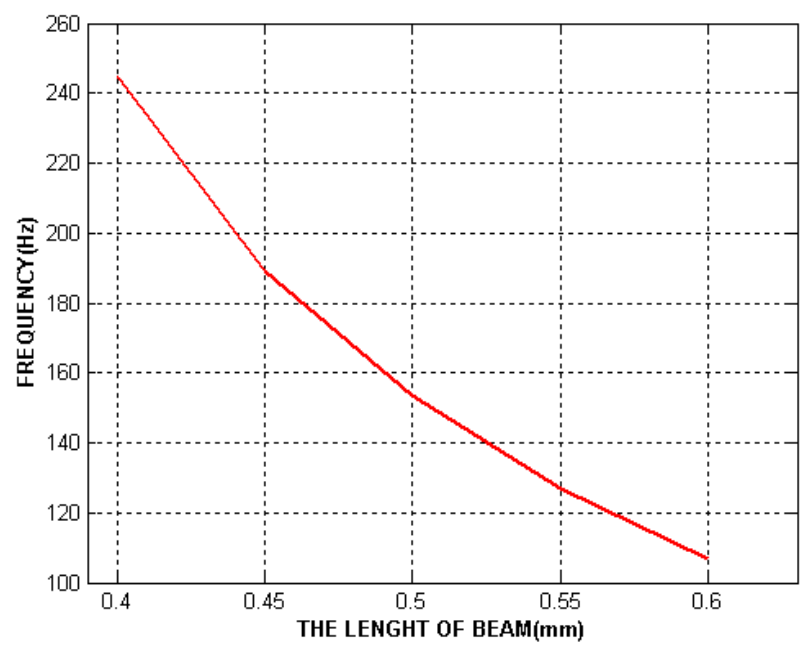

Fig 5 basic natural frequency of beam with $\mathrm{a}=\mathrm{o}$ for different $\mathrm{L}$

Then, the length of the beam $L$ should be increased if it is required to decrease the basic natural frequency for the zero acceleration $a=0$.

From the below Tables and the below Figures, we can get the following results: The variation of the basic natural frequency of the beam decreased with increasing the length of the beam, while the relative variation (sensitivity) of the basic natural frequency increases with increasing the length of the beam for the given parameters of the E-type round diaphragm

The variation of the basic natural frequency of the beam and the relative variation (sensitivity) of the basic natural frequency of the beam is increased with increasing the outer radius of the E-type round diaphragm as the inner radius of the E-type round diaphragm is constant.

The variation of the basic natural frequency of beam 2 is more than that of beam1 And the above tendency decreases with increasing the ratio $\mathrm{B} / \mathrm{A}$ and decreases with increasing the length of the beam. [8,9]

Table 2 The variation frequency $(\mathrm{Hz})$ of Beam 1 for the different $A, L$

\begin{tabular}{|l|c|c|c|c|c|}
\hline \multirow{3}{*}{$A * 10^{-3} m$} & \multicolumn{5}{|c|}{ THE LENGTH OF THE BEAM $\left(L \times 10^{-3} \mathrm{~m}\right)$} \\
\cline { 2 - 6 } & 0.4 & 0.45 & 0.50 & 0.55 & 0.60 \\
\hline 3.5 & 4354 & 4123 & 3790 & 3654 & 3498 \\
\hline 3.6 & 4489 & 4240 & 3878 & 3719 & 3567 \\
\hline 3.7 & 4521 & 4387 & 4115 & 3968 & 3811 \\
\hline 3.8 & 4611 & 4456 & 4343 & 4190 & 4047 \\
\hline
\end{tabular}

Table 3 The variation frequency $(\mathbf{H z})$ of Beam2 for the different $A, L$

\begin{tabular}{|l|c|c|c|c|c|}
\hline \multirow{3}{*}{$A * 10^{-3} m$} & \multicolumn{5}{|l|}{ THE LENGTH OF THE BEAM $\left(L \times 10^{-3} \mathrm{~m}\right)$} \\
\cline { 2 - 6 } & 0.4 & 0.45 & 0.50 & 0.55 & 0.60 \\
\hline 3.5 & -4867 & -4732 & -4587 & -4310 & -4165 \\
\hline 3.6 & -5032 & -4898 & -4778 & -4521 & -4282 \\
\hline 3.7 & -5499 & -5389 & -5181 & -4942 & -4679 \\
\hline 3.8 & -5921 & -5765 & -5594 & -5352 & -5083 \\
\hline
\end{tabular}


Simulation of the different position on the E-type round diaphragm and the length of the beam,

Table 4The relative frequency variation (sensitivity) of Beam1 for the different $A, L$

\begin{tabular}{|l|c|c|c|c|c|}
\hline \multirow{3}{*}{$A * 10^{-3} m$} & \multicolumn{5}{|l|}{ THE LENGTH OF THE BEAM $\left(L \times 10^{-3} \mathrm{~m}\right)$} \\
\cline { 2 - 6 } & 0.4 & 0.45 & 0.50 & 0.55 & 0.60 \\
\hline 3.5 & $1.778 \%$ & $2.150 \%$ & $2.487 \%$ & $2.879 \%$ & $3.279 \%$ \\
\hline 3.6 & $1.834 \%$ & $2.240 \%$ & $2.525 \%$ & $2.930 \%$ & $3.345 \%$ \\
\hline 3.7 & $1.847 \%$ & $2.319 \%$ & $2.680 \%$ & $3.127 \%$ & $3.573 \%$ \\
\hline 3.8 & $1.884 \%$ & $2.356 \%$ & $2.828 \%$ & $3.301 \%$ & $3.795 \%$ \\
\hline
\end{tabular}

Table 5 The relative frequency variation (sensitivity) of Beam2 for the different $A, L$

\begin{tabular}{|l|l|l|l|l|c|}
\hline \multirow{2}{*}{$A * 10^{-3} m$} & \multicolumn{5}{|c|}{ THE LENGTH OF THE BEAM $\left(L \times 10^{-3} \mathrm{~m}\right)$} \\
\cline { 2 - 6 } & 0.4 & 0.45 & 0.50 & 0.55 & 0.60 \\
\hline 3.5 & & & & & \\
\hline 3.6 & $-1.988 \%$ & $-2.501 \%$ & $-2.987 \%$ & $-3.396 \%$ & $-3.905 \%$ \\
\hline 3.7 & $-2.056 \%$ & $-2.601 \%$ & $-3.112 \%$ & $-3.562 \%$ & $-4.015 \%$ \\
\hline 3.8 & $-2.246 \%$ & $-2.849 \%$ & $-3.374 \%$ & $-3.894 \%$ & $-4.387 \%$ \\
\hline
\end{tabular}

Table 6 The variation frequency $\mathbf{( H z )}$ of Beam1 for the different $A, L$

\begin{tabular}{|c|c|l|l|l|c|}
\hline \multirow{2}{*}{$A * 10^{-3} m$} & \multicolumn{5}{|c|}{ THE LENGTH OF THE BEAM $\left(L \times 10^{-3} \mathrm{~m}\right)$} \\
\cline { 2 - 6 } & 0.4 & 0.45 & 0.50 & 0.55 & 0.60 \\
\hline 3.5 & -4277 & -4167 & -3822 & -3755 & -3532 \\
\hline 3.6 & -4310 & -4245 & -3987 & -3858 & -3716 \\
\hline 3.7 & -4576 & -4378 & -4234 & -4114 & -3980 \\
\hline 3.8 & -4789 & -4567 & -4489 & -4364 & -4236 \\
\hline & & & & \\
\hline
\end{tabular}

Table 7 The variation frequency $(\mathrm{Hz})$ of Beam2 for the different $A, L$

\begin{tabular}{|l|l|l|l|l|l|}
\hline \multirow{2}{*}{$A * 10^{-3} m$} & \multicolumn{5}{l}{ THE LENGTH OF THE BEAM $\left(L \times 10^{-3} \mathrm{~m}\right)$} \\
\cline { 2 - 6 } & 0.4 & 0.45 & 0.50 & 0.55 & 0.60 \\
\hline 3.5 & 5076 & 4954 & 4312 & 4211 & 4034 \\
\hline 3.6 & 5134 & 5099 & 4620 & 4342 & 4270 \\
\hline 3.7 & 5376 & 5177 & 4994 & 4719 & 4452 \\
\hline 3.8 & 5743 & 5470 & 5378 & 5085 & 4814 \\
\hline
\end{tabular}

Table 8 The relative frequency variation (sensitivity) of Beam1 for the different $A, L$

\begin{tabular}{|l|l|l|l|l|l|}
\hline \multirow{2}{*}{$A * 10^{-3} m$} & \multicolumn{5}{l}{} \\
\cline { 2 - 6 } & 0.4 & 0.45 & 0.50 & 0.55 & 0.60 \\
\hline 3.5 & $-1.747 \%$ & $-2.203 \%$ & $-2.487 \%$ & $-2.964 \%$ & $-3.311 \%$ \\
\hline 3.6 & $-1.761 \%$ & $-2.244 \%$ & $-2.596 \%$ & $-3.040 \%$ & $-3.484 \%$ \\
\hline 3.7 & $-1.869 \%$ & $-2.314 \%$ & $-2.757 \%$ & $-3.242 \%$ & $-3732 \%$ \\
\hline 3.8 & $-1.956 \%$ & $-2.414 \%$ & $-2.923 \%$ & $-3.439 \%$ & $-3.972 \%$ \\
\hline
\end{tabular}

Table 9 The relative frequency variation (sensitivity) of Beam2 for the different $A, L$

\begin{tabular}{|l|l|l|l|l|l|}
\hline \multirow{2}{*}{$A * 10^{-3} m$} & \multicolumn{5}{|l|}{ THE LENGTH OF THE BEAM $\left(L \times 10^{-3} \mathrm{~m}\right)$} \\
\cline { 2 - 6 } & 0.4 & 0.45 & 0.50 & 0.55 & 0.60 \\
\hline 3.5 & $2.073 \%$ & $2.619 \%$ & $2.808 \%$ & $3.317 \%$ & $3.782 \%$ \\
\hline 3.6 & $2.097 \%$ & $2.695 \%$ & $3.009 \%$ & $3.421 \%$ & $3.826 \%$ \\
\hline 3.7 & $2.196 \%$ & $2.737 \%$ & $3.252 \%$ & $3.718 \%$ & $4.175 \%$ \\
\hline 3.8 & $2.346 \%$ & $2.892 \%$ & $3.502 \%$ & $4.007 \%$ & $4.514 \%$ \\
\hline
\end{tabular}




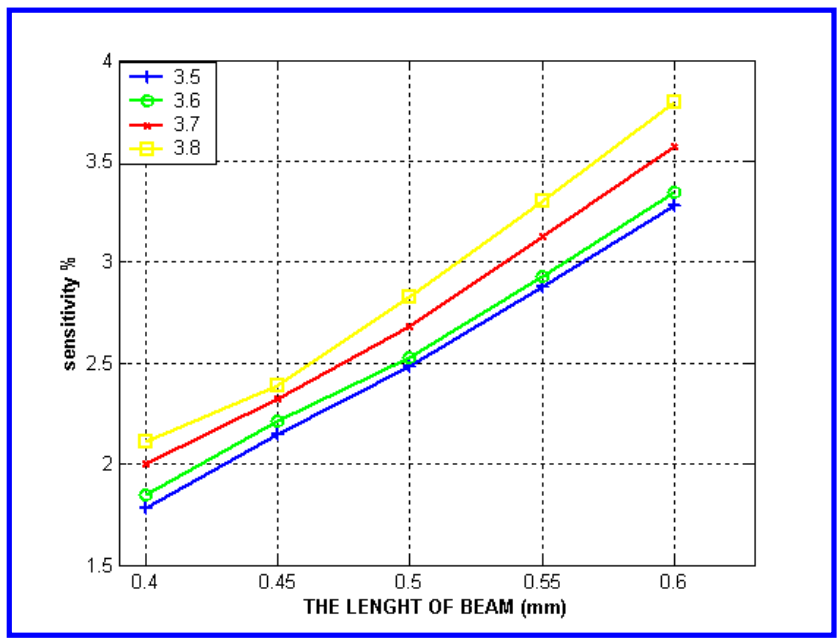

Fig 6 the relationship between the relative frequency variation (sensitivity) of beam 1 and the length of beam

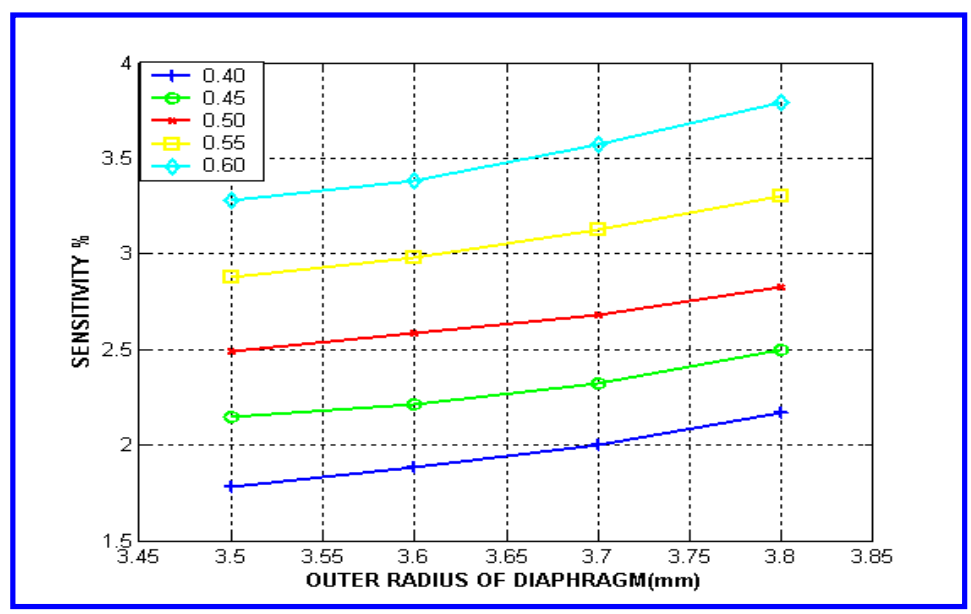

Fig 7 the relationship between the relative frequency variation (sensitivity) of beamland the outer radius of diaphragm A

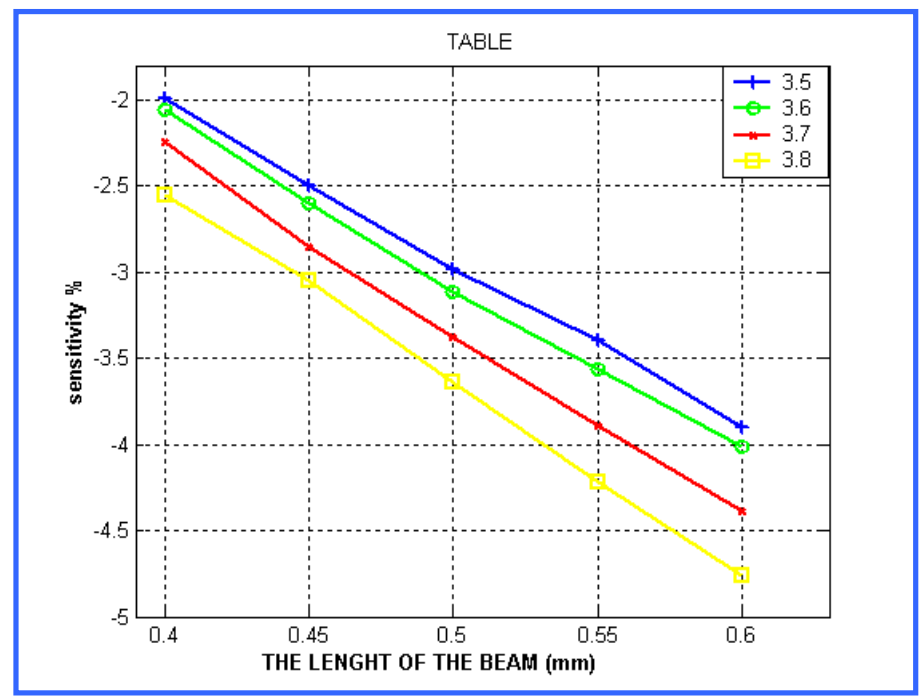

Fig 8 the relationship between the relative frequency variation (sensitivity) of beam2 and the length of beam 


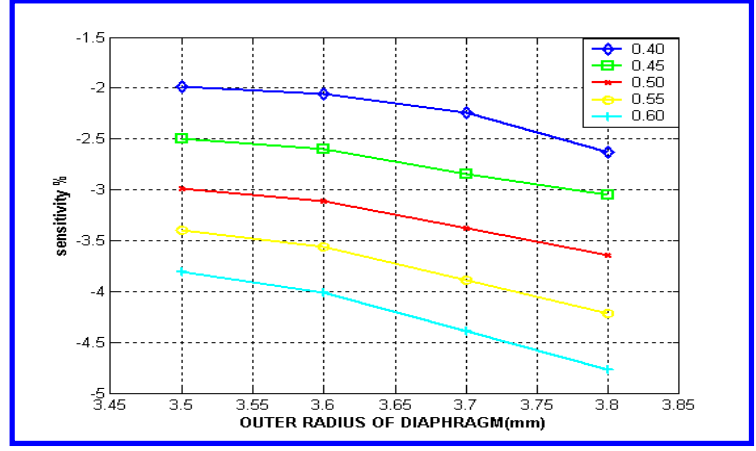

Fig 9 the relationship between the relative frequency variation (sensitivity) of beam2 and the outer radius of diaphragm

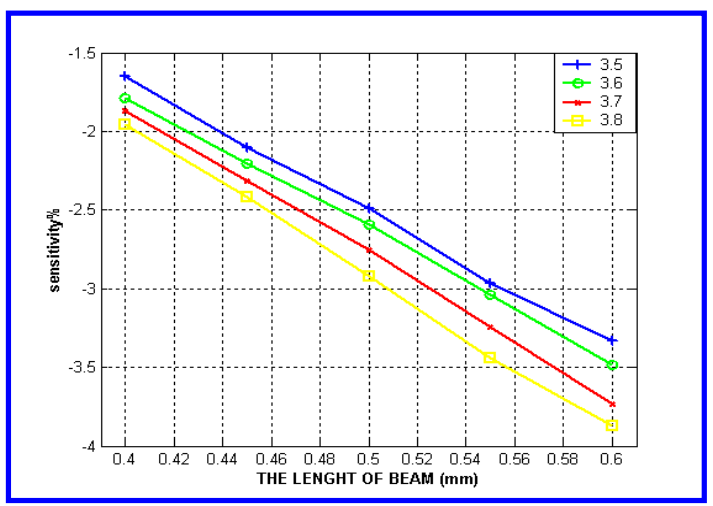

Fig 10 the relationship between the relative frequency variation (sensitivity) of beam 1 and the length of beam

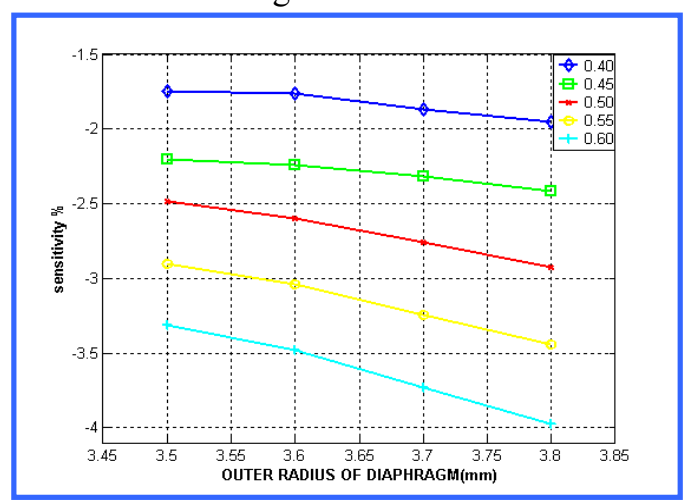

Fig 11 the relationship between the relative frequency variation (sensitivity) of beam 1 and the outer radius of diaphragm $\mathrm{A}$

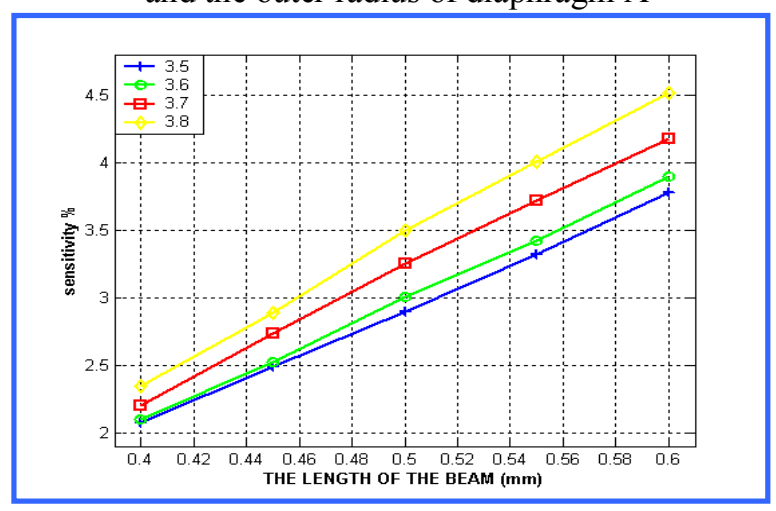

Fig 12 the relationship between the relative frequency variation (sensitivity) of beam2 and the length of beam 


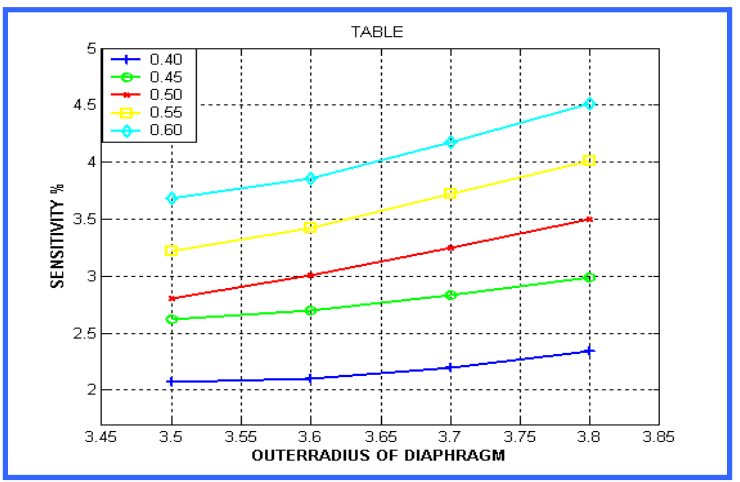

Fig 13 the relationship between the relative frequency variation (sensitivity) of beam2 and the outer radius of diaphragm $\mathrm{A}$

\section{Conclusion}

The modeling and simulation for a novel resonant silicon micro-sensor are carried out in this thesis. The elementary sensing component of the micro-sensor is the E-type round diaphragm and its final sensing component is the beam resonator which is attached to the E-type round diaphragm.

The sensor is made of silicon, $\mathrm{E}=1.3 \times 10^{11} \mathrm{pa}, \rho=2.33 \times 10^{3} \mathrm{~kg} / \mathrm{m}^{3}, \mu=0.17$. The inner radius is as $\mathrm{B}=1.4 \times 10^{-3} \mathrm{~m}$ and the thickness of the E-type round diaphragm is as $H=30 \times 10^{-6} \mathrm{~m}$. The width and thickness of the beam are $b=50 \times 10^{-6} \mathrm{~m}$ and $h=5 \times 10^{-6} \mathrm{~m}$. Assuming the measured acceleration $a \in(-100,+100) \mathrm{m} / \mathrm{s}^{2}$, corresponding inertial force are

$F \in(+1.300,-1.300) \times 10^{-2} N$, to $F \in(+1.304,-1.304) \times 10^{-2} N$ as the outer radius of the Etype round diaphragm are $A=(3.5,3.6,3.7,3.8) \times 10^{-3} \mathrm{~m}$ and the frequencies of the beam are $f(0)=$ $(244745,189134,153561,126914,106648) \mathrm{Hz}$ for measured acceleration $a=0$ as the length of the beam $\mathrm{L}=$ $(400,450,500,550,600) \times 10^{-6} \mathrm{~m}$ respectively.

Then the simulation results are as follows:

the frequency range for Beam1 is $(149327 \sim 157675) \mathrm{Hz}$ which located at the outer edge the measured acceleration as within $(-100,+100) \mathrm{m} / \mathrm{s}^{2}$

the frequency range for Beam2 is $(158555 \sim 148380) \mathrm{Hz}$ which located at the inner edge the measured acceleration as within $(-100,+100) \mathrm{m} / \mathrm{s}^{2}$

In addition to the variation and relative variation or the sensitivity of the basic natural frequency of the beam 1 $\Delta f\left(-100 \mathrm{~m} / \mathrm{s}^{2}\right)=-4234 \mathrm{~Hz}, \Delta f\left(+100 \mathrm{~m} / \mathrm{s}^{2}\right)=4115 \mathrm{~Hz}, \beta\left(-100 \mathrm{~m} / \mathrm{s}^{2}\right)=-2.757 \%, \beta\left(+100 \mathrm{~m} / \mathrm{s}^{2}\right)=2.680 \%$, $f(0)=153561 \mathrm{~Hz}$. And the variation and relative variation or the sensitivity of the basic natural frequency of the beam $\Delta f\left(-100 \mathrm{~m} / \mathrm{s}^{2}\right)=4994 \mathrm{~Hz}, \Delta f\left(+100 \mathrm{~m} / \mathrm{s}^{2}\right)=-5181 \mathrm{~Hz}, \beta\left(-100 \mathrm{~m} / \mathrm{s}^{2}\right)=3.252 \%$

$\beta\left(+100 \mathrm{~m} / \mathrm{s}^{2}\right)=-3.374 \% f(0)=153561 \mathrm{~Hz}$. Moreover, as the differential frequency output of the above accelerometer between Beam1 and Beam2 is $(-9228,9295) \mathrm{Hz}$

\section{References}

[1] P. Parsons, A. Glendinning and D. Angelidis, "Resonator Sensor for High AccuracyPressure Measurement Using Silicon Technology," Schlumberger Industries Aerospace.Transducer Division, IEEE AES Magazine, pp. 45-48, July 1992.

[2] G.Gautschi chapter 9, piezoelectric Sensor (2001) 731-8621-g27z .

[3] Shangchun Fan, Guangyu Liu, Man Hyuna Lee, Finite-element modeling and simulation on a novel microstructure silicon accelerometer with direct frequency output, SPIE Volume 3087, 1997.

[4] H.Baltes,W.Gopel,J.Hesse, "SensorsUpdate”,Volume7,Sensortechnology;Applications; Markets(1998).

[5] Ljubisa Ristic, "Sensor Technology and Devices", Artech House Boston, London (1994).

[6] S. Middelhock, Quo vadis silicon sensor, report, 1996.

[7] S. Middelehoek, Quo vadis silicon sensors \& Actuators, A $41-42$ (1994) 1-8.

[8] H.Baltes,W.Gopel,J.Hesse"SensorsUpdate",Volume4Sensor tchnology; Applications; Markets (1998).

[9] Shangchun Fan, Guangyu Liu, Man Hyuna Lee, Finite-element modeling and simulationon frequency characteristics of the silicon beam resonator attached to an E-type round diaphragm for measuring the concentrated force, Sensors and Actuators, A63 (1997), $169-176$. 